\title{
Association of Positive Results of Single Serum Biomarker with the Clinical Parameters
}

\author{
Lin $\mathrm{Yu}^{1,2}$, Yang Luo ${ }^{3}$, Yisheng $\mathrm{Li}^{1}$, Bitao $\mathrm{Wu}^{1}$, Yan $\mathrm{Ren}^{1}$, Wenqiang Jiang ${ }^{1}$, Jiafu Feng ${ }^{1}$ and Bei Xu*1 \\ ${ }^{1}$ The Clinical Laboratory of Mianyang Central Hospital, China \\ ${ }^{2}$ State Key Laboratory of Biotherapy, West China Medical School, Sichuan University, China \\ ${ }^{3}$ Department of Stomatology, Mianyang Central Hospital, China
}

*Corresponding author: Bei Xu, The Clinical Laboratory of Mianyang Central Hospital, China

\section{ARTICLE INFO \\ Received: 画 May 23, 2019 \\ Published: 慧 May 29, 2019 \\ Citation: Lin Yu, Yang Luo, Yisheng Li, Bitao Wu, Yan Ren, Wenqiang Jiang, Jiafu Feng, Bei Xu. Association of Positive Re- sults of Single Serum Biomarker with the Clinical Parameters. Biomed J Sci \& Tech Res 18(3)-2019. BJSTR. MS.ID.003153.}

Keywords: Serum Tumor Biomarkers; Positive Results; Clinical Parameters

Abbreviations: AFP: Alpha-Fetoprotein; CEA: Carcinoembryonic Antigen; NSE: Neurone Specific Enolase; TPSA: Total Prostate Specific Antigen; HE4: Human Epididymis Secretory Protein 4; CA125: Carbohydrate Antigen 125; SF: Serum Ferritin
ABSTRACT

Background: Many serum biomarkers have been developed to diagnose cancers at an early stage and monitor drug therapy. At present, the most common clinical used serumtumor biomarkers are mainly including Alpha-Fetoprotein (AFP), Carcinoembryonic Antigen (CEA), Neurone Specific Enolase (NSE), Total Prostate Specific Antigen (TPSA), Human Epididymis Secretory Protein 4 (HE4), Carbohydrate Antigen 125 (CA125), CA153, CA19-9, CA72-4 and Serum Ferritin (SF). However, more and more studies have shown that serum markers were poor specific and sensitive in cancer early diagnosis, even among the most correlative cancers.

Methods: In this work, we screened out and statistically analysed the relationship of the positive results and its distribution for all mentioned above serum biomarkers with the clinical parameters including patient's gender, age and disease types.

Results: We found that most single serum biomarker had relatively low association with cancer, even for the most correlative cancer. we also found that the clinical diagnosis corresponding to single biomarker with high serum levels were various in addition to the diagnosis, treatment and prognosis of the most correlative cancer. Besides, there were no direct relationships of certain serum levels of the biomarkers with the type of disease.

Conclusion: In summary, the serum levels of biomarkers cannot be used as the only available criterion for making a clinical decision.

\section{Introduction}

With dramatical population growth and aging worldwide, cancer incidence and mortality become the major public health concern affecting more than millions of people each year [1]. With nearly one-quarter of the people in the world, cancer deaths of China as well as have a severe impact on global cancer trend and burden [2]. As we know, early diagnosis usually could increase the chances of successful treatment which gives great promises for cancer patients [3]. And major advances have been made in screening tissue or blood samples for cancer-specific biomarkers that can detect the cancer as early as possible [4,5]. So far, series of serum biomarkers have been adapted to clinical use for cancer diagnosis, therapy and prognosis monitoring [6]. For example, AFP, a marker for hepatocellular carcinoma, sometimes is used to assess hepatic masses in patients at particular risk for developing hepatic malignancy [7].

CEA is expressed in normal mucosal cells and overexpressed in adenocarcinoma, especially colorectal cancer $[8,9]$. NSE has been evaluated in numbers of malignant tumours, such as lung cancer [10,11]. HE4 [12-14] and CA125 [15,16] have an obvious elevated expression levels in ovarian cancers. CA15-3 is a protein made by a variety of cells, particularly breast cancer cells $[17,18]$. Elevated levels of CA19-9 occur primarily in patients with pancreatic cancers but also have been reported in patients with other malignancies [19-22]. It was reported CA72-4 was the most correlative serum biomarker for the detection of gastric cancer in Chinese population $[23,24]$. Serum Ferritin (SF) can be used as a prognostic biomarker 
for survival in relapsed or refractory metastatic colorectal cancer [25]. With adenocarcinoma in older men, significant PSA elevations have enough specificity to make the diagnosis of prostate cancer, and so on $[26,27]$.

Due to the convenience and non-invasion, those serum biomarkers have been widely used in clinical practice. However, to date, many serum biomarkers have been demonstrated poor specificity and efficacy, even among the most correlative cancers. Because of the overabundance of diagnostic value and clinical significance, it is necessary for doctors and patients to have a thorough understanding of those serum biomarkers [28,29]. The study aimed to provide a comprehensive overview of the relationship of the positive results of serum biomarkers and its distribution with the patient's gender, age and types of disease, and offer more references for clinical practise.

\section{Materials and Methods}

\section{Data}

The serum levels of serum biomarkers referred to the Clinical Laboratory of Mianyang Central Hospital, China, between January 2016 and September 2018 were collected. And only tested results from patients with complete information including gender, age and clinical diagnosis were selected for this study. And serum levels of biomarkers above the upper reference values were defined as positive. Firstly, we summarized all the test results for each tumor marker, and then screened out the positive results of each biomarker. The detailed cases of each tumor marker had been listed in Table 1. All statistical analyses were performed on the positive cases of each tumor marker.

Table 1: The analyzed cases of positive results for each serum biomarker.

\begin{tabular}{|c|c|c|}
\hline Biomarkers & Cases & Positive Cases \\
\hline AFP & 42253 & 3451 \\
\hline CEA & 53552 & 8004 \\
\hline NSE & 487 & 123 \\
\hline TPSA & 16485 & 3241 \\
\hline HE4 & 7102 & 807 \\
\hline CA125 & 25834 & 6557 \\
\hline CA153 & 16010 & 800 \\
\hline CA19-9 & 39937 & 5459 \\
\hline CA72-4 & 1512 & 475 \\
\hline Ferritin (Male) & 6426 & 2665 \\
\hline Ferritin (Female) & 30094 & 2178 \\
\hline
\end{tabular}

\section{Laboratory Methods}

According to the criteria, all serum samples were collected into $5 \mathrm{~mL}$ tubes (BD Vacutainer ${ }^{\circledR}$ SST II Advance tube) in the morning with fasting time 8-14 h. After centrifuged at $3000 \mathrm{rpm}$ for $15 \mathrm{~min}$, serum samples were measured within $4 \mathrm{~h}$. Serum CA72-4 was measured by an electrochemiluminescence immunoassay on Roche Cobas e601 immunoassay analyzer (Roche Diagnostics, Germany).
Serum AFP, CEA, TPSA, HE4, CA125, CA153, CA19-9 and SF were tested by the chemiluminesent micropaticle immunoassay on the ARCHITECT i2000SR immunoassay analyzer (Abbott, USA). Serum NSE was detected by the LIAISON XL chemiluminescence immune analyzer. The recommended reference intervals of those serum biomarkers were shown in Table 2.

Table 2: The reference intervals for biomarkers.

\begin{tabular}{|c|c|c|}
\hline Biomarker & Reference interval & Unit \\
\hline AFP & $0.00-8.78$ & $\mathrm{IU} / \mathrm{mL}$ \\
\hline CEA & $0.00-5.00$ & $\mathrm{ng} / \mathrm{mL}$ \\
\hline NSE & $0.00-18.30$ & $\mathrm{\mu g} / \mathrm{L}$ \\
\hline TPSA & $0.00-4.00$ & $\mathrm{ng} / \mathrm{mL}$ \\
\hline HE4 & $0.00-140.00$ & $\mathrm{pmol} / \mathrm{L}$ \\
\hline CA125 & $0.00-35.00$ & $\mathrm{U} / \mathrm{mL}$ \\
\hline CA153 & $0.00-31.30$ & $\mathrm{U} / \mathrm{mL}$ \\
\hline CA19-9 & $0.00-37.00$ & $\mathrm{U} / \mathrm{mL}$ \\
\hline CA72-4 & $5.60-8.20$ & $\mathrm{U} / \mathrm{mL}$ \\
\hline Ferritin (Male) & $21.81-274.66$ & $\mathrm{ng} / \mathrm{mL}$ \\
\hline Ferritin (Female) & $4.63-204.00$ & $\mathrm{ng} / \mathrm{mL}$ \\
\hline
\end{tabular}

\section{Statistical Analyses}

All statistical analyses were performed with SPSS 13.0 for Windows (SPSS, Chicago, IL, USA), and $p<0.05$ was considered statistically significant. The association of tested serum levels of biomarkers with the patient's gender, age and disease types were performed by the Mann-Whitney U test.

\section{Results}

In this study, the positive results of ten kinds of serum-based biomarkers were statistically analysed according to the types of diseases, gender and age as shown as in Figure 1. Firstly, the rates of positive results associated with the diagnosis, treatment or prognosis for the cancer were calculated. The rates above $50 \%$ were respectively from CEA (62.13\%) and CA125 (50.10\%). For NSE and CA19-9 biomarkers, there were comprised $43.09 \%$ and $40.87 \%$, respectively. And for AFP, CA125, HE4, TPSA and SF, there were separately held $28.51 \%, 26.66 \%, 26.02 \%, 17.37 \%$ and $10.26 \%$. As shown, CA72-4 had the lowest proportion, only 4.42\%. Besides, statistical analyse showed that positive results of serum biomarkers also were gender specific. For example, $99.88 \%$ of positive results for TPSA were from elderly male. And CA125 (97.73\%), CA153 (98.00\%) and HE4 (99.50\%) were shown specific for female. Finally, the relationship between positive results of serum-based biomarkers and age were statistically analysed. And the proportion of the positive result of TPSA for male patients whose age exceeded 60 years old was $89.17 \%$. And the proportions for HE4, FEER and CEA biomarkers were $66.91 \%, 61.99 \%$ and $58.87 \%$, respectively. But the percentages for CA19-9, NSE, CA153, AFP, CA125 and CA724 serum markers were relatively low (see Figure 1). 

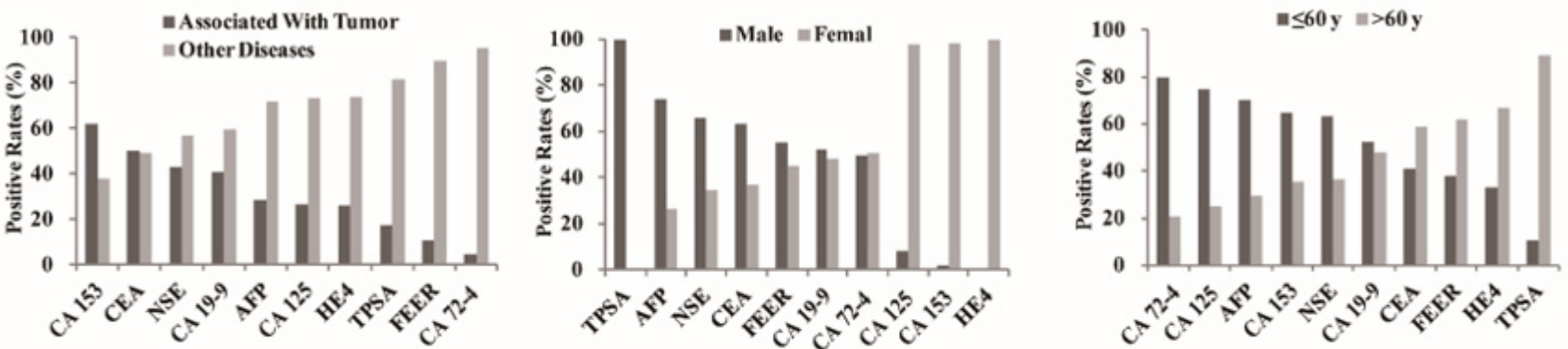

\begin{tabular}{|c|c|c|}
\hline Biomaken & Associated With Tumor (\%) & Other Diseases (\%) \\
\hline CA 153 & 62.13 & 37.87 \\
\hline CE.A & 50.10 & 48.90 \\
\hline NSE & 43.09 & 56.91 \\
\hline CA 19-9 & 40.87 & 59.13 \\
\hline AFP & 28.51 & 71.49 \\
\hline CA 125 & 26.66 & 73.34 \\
\hline HE4 & 26.02 & 73.98 \\
\hline TPSA & 17.37 & 81.63 \\
\hline FEER & 10.26 & 89.74 \\
\hline CA 72-4 & 4.42 & 95.58 \\
\hline
\end{tabular}

\begin{tabular}{|c|c|c|}
\hline Biomaken & Male (\%) & Femal (\%) \\
\hline TPSA & 99.88 & 0.12 \\
\hline AFP & 73.89 & 26.11 \\
\hline NSE & 65.85 & 34.15 \\
\hline CEA & 63.34 & 36.66 \\
\hline FEER & 55.03 & 44.97 \\
\hline CA 19-9 & 52.12 & 47.88 \\
\hline CA 72-4 & 49.47 & 50.53 \\
\hline CA 125 & 7.76 & 97.24 \\
\hline CA 153 & 2.00 & 98.00 \\
\hline HE4 & 0.50 & 99.50 \\
\hline
\end{tabular}

\begin{tabular}{|c|c|c|}
\hline Biomaken & $\leqslant 60 \mathbf{y}(\%)$ & $>60 \mathbf{y}(\%)$ \\
\hline CA 72-4 & 79.39 & 20.63 \\
\hline CA 125 & 74.85 & 25.15 \\
\hline AFP & 70.41 & 29.59 \\
\hline CA 153 & 64.50 & 35.50 \\
\hline NSE & 63.41 & 36.59 \\
\hline CA 19-9 & 52.12 & 47.88 \\
\hline CEA & 41.13 & 58.87 \\
\hline FEER & 38.01 & 61.99 \\
\hline HE4 & 33.09 & 66.91 \\
\hline TPSA & 10.83 & 89.17 \\
\hline
\end{tabular}

Figure 1: Comprehensive overview the association of serum single biomarker with the patient's gender, age and types of disease. The positive cases of AFP, CEA, NSE, TPSA, HE4, CA125, CA153, CA19-9, CA72-4 and SF were screened out and summarized. Among these positive results, the patients were divided according to the clinical parameters (ages, associated with cancer or other diseases, and male or female). Next, the rates of each clinical parameter were calculated, $P<0.05$.

In order to fully understand the relationship between single serum biomarker and diseases, the clinical diagnosis of the positive results of each biomarker were classified and counted. As a result, we found that the various clinical diagnosis corresponding to single serum biomarker with high serum levels includes in addition to the diagnosis, treatment and prognosis of the most correlative cancer. For example, AFP were used as a marker for hepatocellular carcinoma, sometimes to assess cirrhosis or hepatic masses. In our study, we also found that when patients were suffering rectal cancer or gastric cancer, the serum level of AFP marker were high. At the same time, when patients were diagnosed as jaundice or gastrointestinal bleeding or biliary disease or gastritis, the serum levels of AFP were also elevated. CEA was reported a biomarker especially for colorectal cancer. And our statistical analysis discovered that many types of cancers, including gastric cancer, rectal cancer, colon cancer, lung cancer, breast cancer, liver cancer, cardia cancer or prostate cancer, could cause the high level of CEA. In addition to the above cancers, other diseases such as cirrhosis, diabetes, liver mass, intestinal obstruction, coronary heart disease or gastritis could also raise up the serum levels of CEA. Consistent with research reports, NSE was evaluated in lung cancer or neuroblastoma. But we also found the high serum levels of NSE in patients with bacterial pneumonia, lung abscess, lung space, lung infection or diabetes. As we known, TPSA has been used as a marker for prostate cancer. It was interesting that we found that the largest numbers of positive cases were benign prostatic hyperplasia. However, for CA125, the maximum positive cases were adenomyosisn, not ovarian cancers. The other diseases were stomachache, ovarian cyst, menstrual cycle related diseases, breast cancer, uterine fibroids, endometriosis, pleural effusion, gastric cancer, colorectal cancer and coronary heart disease.

Compared with CA125, HE4 may be more specific for ovarian cancers. And our statistical analysis also discovered that cirrhosis, coronary heart disease, lung infection, diabetes, gastrointestinal bleeding, intestinal obstruction and lung cancer could cause the high level of HE4. CA153 was selected as a specific biomarker for breast cancer. In addition to breast cancer, there were other diseases such as ovarian cancer, lung cancer, cirrhosis, lung infection, colorectal cancer, coronary heart disease or cervical cancer with high levels of serum CA153. Statistical analysis for CA19-9, positive results were most likely for gastric cancer, colon cancer and rectal cancer. But we also found the high serum levels for patient suffering jaundice, liver cancer, pancreatic cancer, liver mass, pancreatitis, cirrhosis, diabetes, and so forth. Gastritis may be the main reason for highlevel of serum CA72-4, and other causes were ulcer, gastric cancer, cardia cancer and esophagitis.

As shown, anemia was a common blood disorder for both male and female patients when SF serum level was high above the cutoff value. And for male, serum ferritin concentrations were highly correlated with diseases including liver cancer, liver mass, diabetes, lymphoma, thalassemia, and so on. But for female, serum ferritin concentrations closed with pregnancy, fever, lung infection, and so on (Figure 2). Because a little part of other clinical diagnosis had too few cases, so the positive cases of the clinical diagnosis for each serum biomarker were not complete and the rates of summarized different clinical diagnosis also had been listed in Figure 2 . 


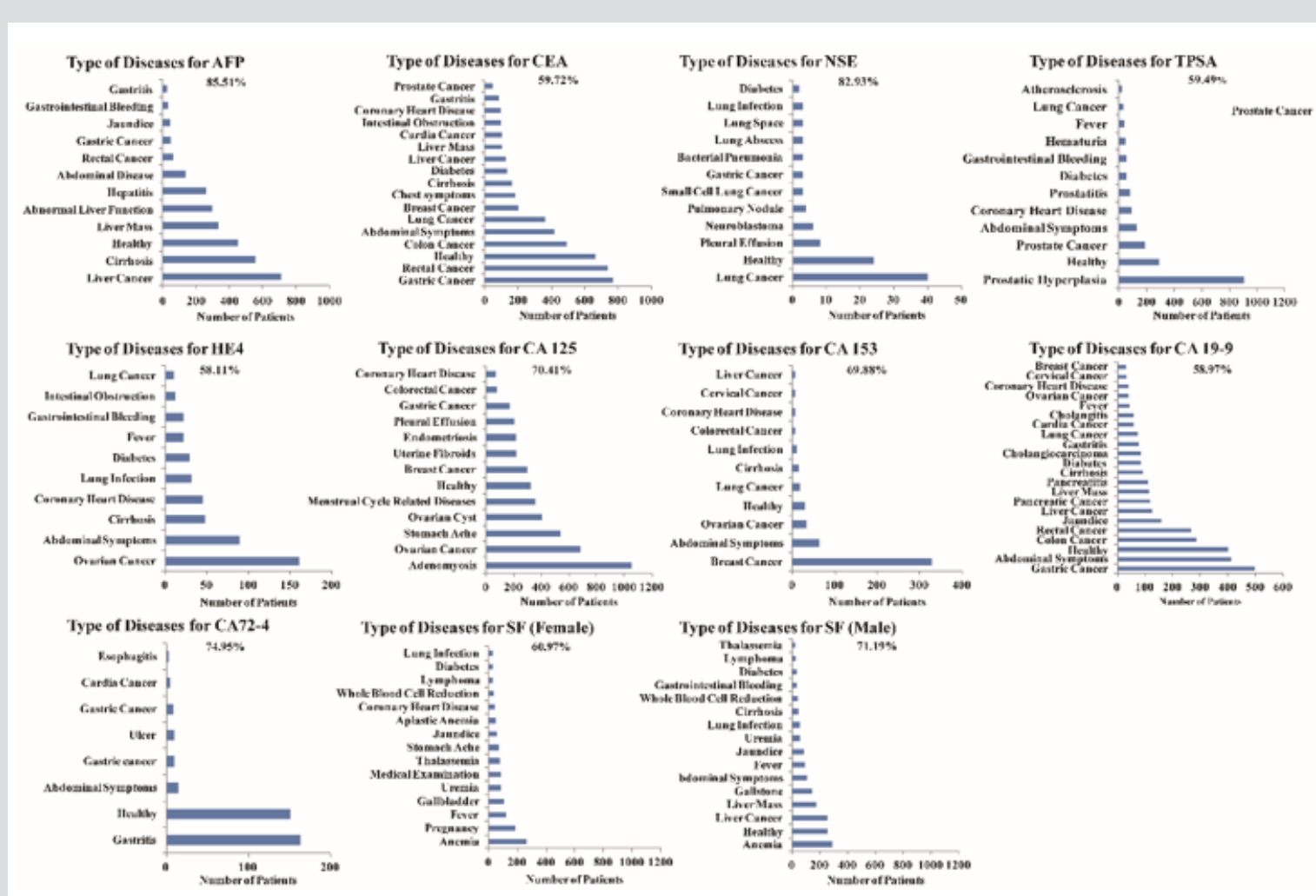

Figure 2: The detailed types of diseases for each single serum biomarker. To understand the relationship between single serum biomarker and diseases, the clinical diagnosis of the positive results of each biomarker were classified and counted. And we also summarized the number of patients with the same diagnosis and counting the types of diseases as much as possible showed by blue bar graph and the partition of summarized different diagnosis was showed in the graph, $P<0.05$.

Combined with the clinical diagnosis of the patients, the positive results of each biomarker were divided into three categories as shown in the Figure 3. And the distributions for the results of the three categories were statistically analysed to further explore the relationship between certain values and the types of diseases.
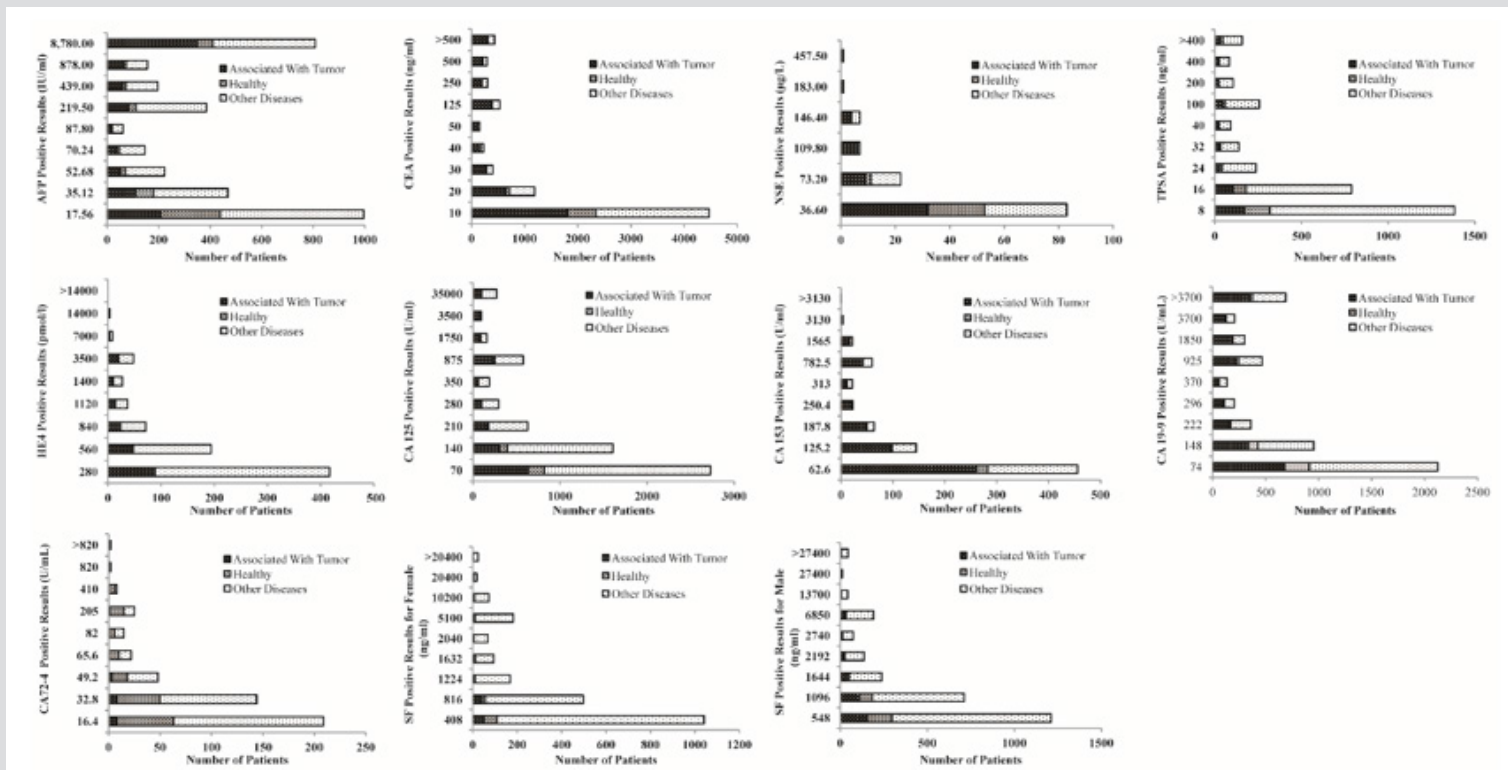

Figure 3: The distribution of the positive results from serum tumor biomarker. The positive results of ten kinds of biomarkers were divided as three categories including associated with tumor, healthy and other diseases which were represented by black, gray and white bar graphs. And the distributions of the results of each single biomarker according to the three categories were showed, $P<0.05$. 
From the results, the cases of three categories for each biomarker were mainly distributed in twice the reference interval and the results from the patents associated with tumor were distributed in each reference interval. And the certain tested results of the biomarkers were not directly related to the type of disease.

\section{Discussion}

In this study, most single serum biomarkers had relatively low diagnostic value for cancer, even for the most correlative cancer. For example, CA72-4 have the lowest proportion (4.42\%) and gastritis was the main reason for high-level of serum CA72-4. And the maximum positive cases for CA125 were adenomyosis instead of ovarian cancers and the largest positive results of TPSA were related with patients were suffering benign prostatic hyperplasia. For CA19-9, positive results were most likely for gastric cancer, colon cancer and rectal cancer. At the same time, the diverse disease types including healthy people were responsible for high serum levels of single serum biomarker. Besides, there were no direct relationships of certain values of the biomarkers with the type of disease. Therefore, combined with multiple serum biomarkers can potentially capture more fully about diseases and provide more comprehensive clinical information.

In summary, in some situations, the serum levels above the cut-off value of biomarkers cannot be used as the only available criterion for making a clinical decision. Clinicians should take more account of the positive results of biomarkers as well as patient's other information including gender, age and disease types and determine whether and in what setting a biomarker can and should be used for patient care, or whether additional evaluation is required before it can be incorporated into routine medical practice. The current studies provide valuable references for a comprehensive understanding of the relationship of those biomarkers with types of diseases. At the same time, more systematic and in-depth researches are deeply needed to improve the diagnostic value and clinical significance of serum biomarkers because of its convenience and non-invasion. In the future, the trend will be to explore more specific and sensitive genomic, proteomic, and epigenetic biomarkers and simultaneously measure those biomarkers by more specific and sensitive techniques [30-32].

\section{Conflicts of Interest}

There are no conflicts of interest regarding the publication of this paper.

\section{Funding Statement}

This study was supported by Sichuan Provincial Health and Family Planning Commission Research Project (No.18PJ011).

\section{References}

1. Bray F, Ferlay J, Soerjomataram I, Siegel RL, Torre LA, et al. (2018) Global cancer statistics 2018: GLOBOCAN estimates of incidence and mortality worldwide for 36 cancers in 185 countries. CA Cancer J Clin 2018: 68 (6): 394-424
2. (2018) World Health Organization. Global Health Observatory. Geneva: World Health Organization; 2018

3. Smith RA, Cokkinides V, Eschenbach ACV, et al. (2002) American cancer society guidelines for the early detection of cancer. CA Cancer J Clin 52 (1): 8-22.

4. Srinivas PR, Kramer BS, Srivastava S (2001) Trends in biomarker research for cancer detection. Lancet Oncol 2 (11): 698-704.

5. Sidransky D (2002) Emerging molecular markers of cancer. Nature Rev Cancer 2 (3): 210-219.

6. Kiviat NB, Critchlow CW (2002) Novel approaches to identification of biomarkers for detection of early stage cancer. Dis Markers 18 (2): 7381.

7. Han K, Tzimas GN, Barkun JS, Peter Metrakos, Jean I Tchervenkov, Nir Hilzenrat, et al. (2007) Preoperative alpha-fetoprotein slope is predictive of hepatocellular carcinoma recurrence after liver transplantation. Can J Gastroenterol 21 (1): 39-45.

8. Nakagoe T, Sawai T, Ayabe H, Nakazaki T, Ishikaw H, Hatano K, et al. (2001) Prognostic value of carcinoembryonic antigen (CEA) in tumor tissue of patients with colorectal cancer. Anticancer Res 21 (4B): 30313036.

9. Grunnet M, Sorensen JB, Carcinoembryonic antigen (CEA) as tumor marker in lung cancer. Lung Cancer 76 (2): 138-143.

10. Ferrigno D, Buccheri G, Giordano C (2003) Neuron-specific enolase is an effective tumour marker in non-small cell lung cancer (NSCLC). Lung Cancer 41 (3): 311-320.

11. Oya Y, oshida TY, Uemura T, Murakami Y, Inaba Y, et al. (2018) Serum ProGRP and NSE levels predicting small cell lung cancer transformation in a patient with ALK rearrangement-positive non-small cell lung cancer: A case report. Oncol Lett 16 (4): 4219-4222.

12. Hellström I, Raycraft J, Hayden Ledbetter M, Ledbetter JA, Schummer $M$, et al. (2003) The HE4 (WFDC2) protein is a biomarker for ovarian carcinoma. Cancer Res 63(13): 3695-3700.

13. Simmons AR, Baggerly K, Bast RCJ (2013) The emerging role of HE4 in the evaluation of epithelial ovarian and endometrial carcinomas. Oncology 27(6): 548-556.

14. Anastasi E, Granato T, Falzarano R, Paola Storelli, Adele Ticino, et al. (2013) The use of HE4, CA125 and CA72-4 biomarkers for differential diagnosis between ovarian endometrioma and epithelial ovarian cancer. J Ovarian Res 6(1): 44.

15. Markmann S, Gerber B, Briese V (2007) Prognostic value of CA125 levels during primary therapy. Anticancer Res 27(4A): 1837-1839.

16. Canney PA, Moore M, Wilkinson PM, et al. (1984) Ovarian cancer antigen CA125: a prospective clinical assessment of its role as a tumour marker Br J Cancer 50 (6): 765-769.

17. Incoronato M, Mirabelli P, Catalano O, Aiello M, Parente C, et al. (2014) CA15-3 is a useful serum tumor marker for diagnostic integration of hybrid positron emission tomography with integrated computed tomography during follow-up of breast cancer patients. BMC Cancer 21 (14): 356.

18. Kazarian A, Blyuss O, Metodieva G, Gentry-Maharaj A, Ryan A et al. (2017) Testing breast cancer serum biomarkers for early detection and prognosis in pre-diagnosis samples. Br J Cancer 116 (4): 501-508.

19. Ballehaninna UK, Chamberlain RS (2011) Serum CA19-9 as a Biomarker for Pancreatic Cancer-A Comprehensive Review. Indian J Surg Oncol 2 (2): 88-100.

20. Ballehaninna UK, Chamberlain RS (2012) The clinical utility of serum CA19-9 in the diagnosis, prognosis and management of pancreatic adenocarcinoma: An evidence based appraisal. J Gastrointest Oncol 3(2): 105-119. 
21. Zhong W, Yu Z, Zhan J (2015) Association of serum levels of CEA, CA19-9 CA125, CYFRA21-1 and CA72-4 and disease characteristics in colorectal cancer. Pathol Oncol Res 21: 83-95.

22. Molina R, Marrades RM, Augé JM, et al. (2016) Assessment of a combined panel of six serum tumor markers for lung cancer. Am J Respir Crit Care Med 193 (4): 427-437.

23. Mariampillai A I, Cruz JPD, Suh J, Sivapiragasam A, Nevins K, et al. (2017) Cancer Antigen 72-4 for the Monitoring of Advanced Tumors of the Gastrointestinal Tract, Lung, Breast and Ovaries. Anticancer Res 37 (7): 3649-3656.

24. Zou L, Qian J (2014) Decline of serum CA724 as a probable predictive factor for tumor response during chemotherapy of advanced gastric carcinoma. Chin J Cancer Res 26 (4): 404-409.

25. Sookyung L, Anna S, Wankyu E (2016) Serum ferritin as a prognostic biomarker for survival in relapsed or refractory metastatic colorectal cancer. J Cancer 7 (8): 957-964.

26. Gann PH, Hennekens CH, Stampfer MJ (1995) A prospective evaluation of plasma prostate-specific antigen for detection of prostatic cancer. JAMA 273 (4): 289-294.

\section{ISSN: 2574-1241}

DOI: 10.26717/BJSTR.2019.18.003153

Bei Xu. Biomed J Sci \& Tech Res

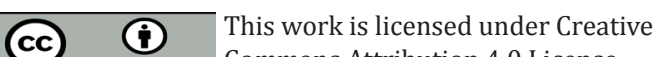

Submission Link: https://biomedres.us/submit-manuscript.php
27. Kontos CK, Adamopoulos PG, Scorilas A (2015) Prognostic and predictive biomarkers in prostate cancer. Expert Rev Mol Diagn 15 (12): 1567-1576.

28. Sturgeon C (2002) Practice guidelines for tumor marker use in the clinic. Clin Chem 48 (8): 1151-1159.

29. Konforte D, Diamandis EP (2013) Is early detection of cancer with circulating biomarkers feasible. Clin Chem 59 (1): 35-37.

30. Gaudreau PO, Stagg J, Soulières D, Fred Saad (2016) The present and future of biomarkers in prostate cancer: proteomics, genomics, and immunology advancements. Biomark Cancer 5(6): 15-33.

31. Kalia M (2015) Biomarkers for personalized oncology: recent advances and future challenges. Metabolism 64 (3): 16-21.

32. Duffy MJ (2017) Clinical use of tumor biomarkers: An overview. Klin Biochem Metab 25(46): 157-161.

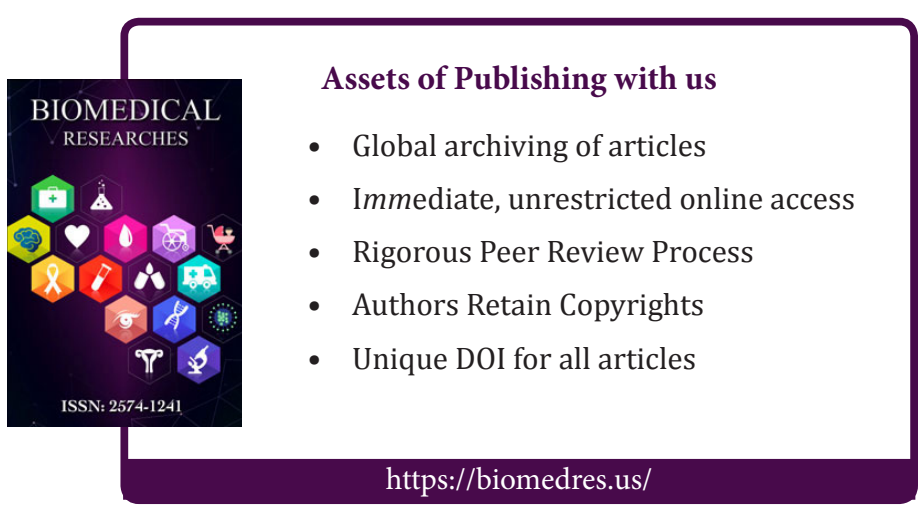

